\title{
Large Scale Patterns on the Solar Surface Indicated by Microwave Observations
}

\author{
B. Vršnak ${ }^{1}$, S. Pohjolainen ${ }^{2}$, H. Teräsranta ${ }^{2}$, S. Urpo ${ }^{2}$, \\ R. Brajša ${ }^{1}$, V. Ruždjaki ${ }^{1}$ A. Schroll ${ }^{3}$, S. Jurač ${ }^{1}$ \\ ${ }^{1}$ Hvar Observatory, 58450 Hvar, Yugoslavia \\ ${ }^{2}$ Metsähovi Radio Research Station, 02540 Kylmälä, Finland \\ ${ }^{3}$ Sonnenobservatorium Kanzelhöhe, 9520 Sattendorf, Austria
}

\begin{abstract}
A large set of observations of the Sun at $37 \mathrm{GHz}$ is analysed. An association of $99 \%$ is found between the regions of brightness temperature depression and the magnetic field inversion lines. Observations indicate a possible existence of "giant cells" with duration of 1-2 years and a longitudinal extension up to $90^{\circ}$.
\end{abstract}

\section{Introduction}

Observations of the Sun in the millimetric range disclose two categories of features which are not related to active regions. These are the areas of low amplitude brightness temperature depressions (Schmahl et al., 1981) and low amplitude enhancements occurring in polar regions (Urpo and Pohjolainen, 1987).

We present here a study of low temperature regions (LTRs) based on a large set of observations measured at $37 \mathrm{GHz}$ at the Metsähovi Radio Research Station during the years $1979-1989$. The beam size of the $13.7 \mathrm{~m}$ radio telescope is 2.4 arc min at $8 \mathrm{~mm}$ wavelength. The temperature resolution is typically better than $1 \%$ of the quiet Sun brightness temperature (estimated as $7800 \mathrm{~K}$ at $8 \mathrm{~mm}$ ).

\section{Results}

When the positions of the temperature minima within LTRs are compared with the inversion lines of the inferred magnetic fields, Fig. 1, one finds that $99 \%$ of the LTRs are lying on the inversion lines presented in the Solar Geophysical Data (SGD) or in the Solnechnie Dannye Byulleten (SDB). Comparing the positions of LTRs with the positions of filaments (Meudon Observatory, courtesy Z. Mouradian) one finds a $60 \%$ association. 
The latitudinal distribution of the LTRs discloses two belts of LTRs which embed a belt of active regions of the actual cycle, Fig. 2. A cradle in the LTR distribution associated with the forthcoming cycle is formed at latitudes of about $50^{\circ}$, already at the maximum of previous cycle.

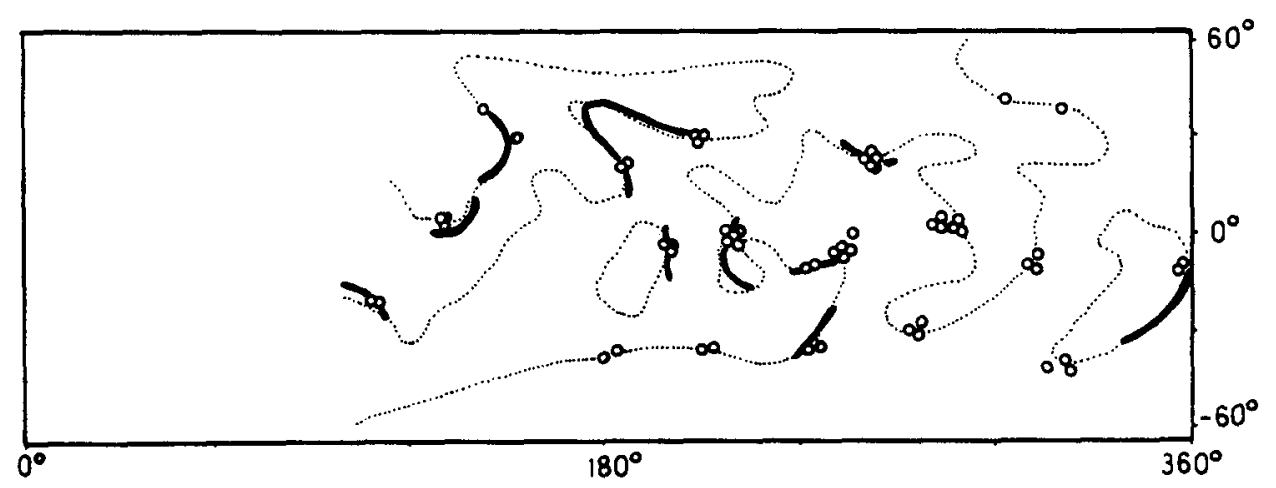

Fig. 1. Association of LTRs (circles) with the SGD magnetic inversion lines (dotted line) and filaments (bold lines).

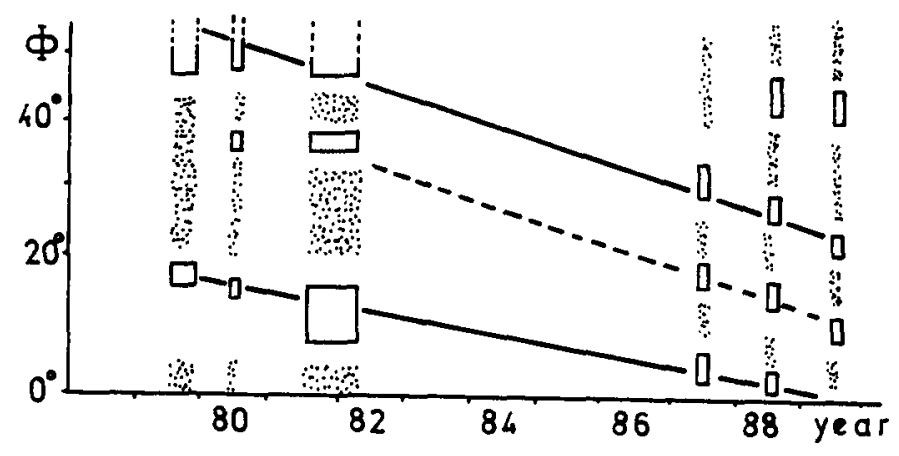

Fig. 2. Latitudinal distribution of microwave features (dotted - LTR belts; rectangles minima in LTR distribution). Active region belts of the Cycles 21 and 22 (bold lines) are separated by a broken line.

In Fig. 3 the superposition of LTRs in the period from May 1979 to May 1980 is presented. The distribution of LTRs indicates a possible existence of large-scale cell-like patterns which are arbitrarily outlined by a thin line. Such patterns could also be found in other analysed periods. The typical longitudinal and latitudinal extensions are $60^{\circ}-90^{\circ}$ and $30^{\circ}-40^{\circ}$, respectively. The life-time could be estimated 
to be 1-2 years. In order to check the reliability of these patterns we compared our results with the results based on magnetographic data (Bumba, 1987). In Fig. 3 the positions of the cell centers taken from Bumba's study are presented, and there is a very good correspondence.

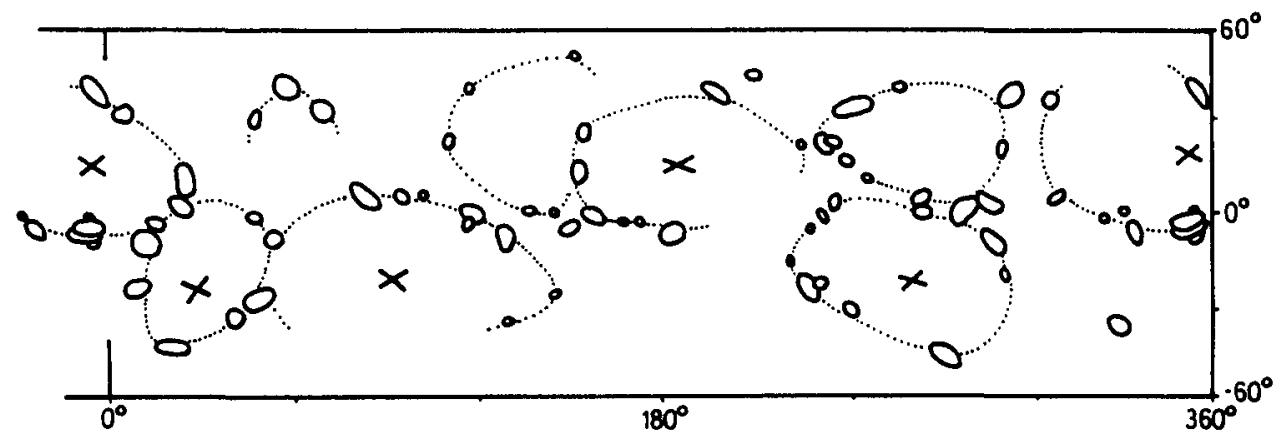

Fig. 3. Superposition of LTRs observed from May 1979 to May 1980. The "cells" are sketched by dotted lines and the centers of Bumba's cells are indicated by crosses.

\subsection{Discussion and conclusions}

The LTRs are directly related to the magnetic inversion lines, while the association with filaments could be accidental since filaments occupy a considerable fraction of the inversion line length. The LTRs represent a large-scale phenomenon related to the evolution of the solar cycle. Although a belt of LTRs corresponding to the polar crown filament belt could be detected on the microwave maps, it was not possible to find any poleward migration as LTRs are rarely detected at latitudes higher than $50^{\circ}$.

Finally, let us note that we can not claim that our results undoubtedly indicate the existence of giant cells but we would like to stress the very good correspondence with the results obtained by Bumba (1987).

\section{References}

Bumba, V.: 1987, Bull. Astron. Inst. Czech. 38, 92

Schmahl, E.J., Bobrowsky, M., Kundu, M.R.: 1981, Solar Phys. 71, 311

Urpo, S., Pohjolainen, S.: 1987, Hvar Obs. Bull. 11, 137 